\title{
Microstructural Defects in Ferroelectrics and Their Scientific Implications
}

\author{
Duo Liu \\ State Key Laboratory of Crystal Materials, \\ Shandong University, Jinan, Shandong, \\ P. R. China
}

\section{Introduction}

Properties of materials are closely linked to their defect structure. Numerous studies have proved that the existence of a small amount of microstructural defects can dramatically change the way of materials behaving in response to external fields. Based on these, various kinds of functional devices have been developed, which have changed the daily life of human beings. Currently, the most important application of defects in industry is probably semiconductor devices intentionally doped with foreign atoms to realize desirable band structures to tune the behaviors of electrons. Defects are also intentionally introduced into metals and insulators to achieve better performances.

Similarly, defects in ferroelectric materials are also extremely important. As a subject that has been investigated for decades, it has been proved that defects and the associated stress and electrical fields could change ferroelectric behaviors such as polarization reversal, domain kinetics, phase transition temperatures, and ferroelectric fatigue. Up to date, numerous studies have been devoted to understanding oxygen vacancies, dislocations, domain walls, voids, and microcracks in ferroelectrics. Actually, almost all aspects of ferroelectric properties are defect-sensitive. For example, doped PZTs could either be "soft" or "hard" with variable coercive fields. Oxygen vacancies play a determinant role on the fatigue process of ferroelectric oxides. Dislocations may hinder the motion of ferroelectric domain walls.

Recent interests on the design and fabrication of nanodevices stem from the distinct and fascinating properties of nanostructured materials. Among those, ferroelectric nanostructures are of particular interests due to their high sensitivity, coupled and ultrafast responses to external inputs [1]. With the decrease of the size of ferroelectric component down to nanoscale, a major topic in modern ferroelectrics is to understand the effects of defects and their evolution [2]. Defects will change optical, mechanical, electrical and electromechanical behaviors of ferroelectrics [3, 4]. However current understanding is limited to bulk and thin film ferroelectrics and is still not sufficiently enough to describe their behaviors at nanoscale. In view of the urgent requirement to integrate ferroelectric components into microdevices and enhanced size-dependent piezoelectricity for nanosized ferroelectric heterostructure, [5] it becomes essential to explore the role of defects in nanoscale ferroelectrics. 
In this Chapter, the author will first discuss the effects associated with different types of defects in $\mathrm{BaTiO}_{3}$, a model ferroelectric, from the point of views of the classical ferroelectric Landau-Ginsberg-Devonshire (LGD) theory. The author will then present some recent progresses made on this area. Among those include 1) critical size for dislocation in $\mathrm{BaTiO}_{3}$ nanocube, 2) (111) twined $\mathrm{BaTiO}_{3}$ microcrystallites and the photochromic effects.

\section{Thermodynamic description of ferroelectrics}

Most important phenomena associated with hysteretic, polarization, domain wall, and phase transition behaviors in ferroelectrics can be described by using the thermodynamic Landau-Ginzburg-Devonshire (LGD) theory. The LGD theory has been demonstrated to be the most powerful tool to understand ferroelectric behaviors especially when the materials are under the influence of external fields (electrical, temperature, and stress) [6, 7].

Most ferroelectric materials undergo a structural phase transition from a high temperature non-ferroelectric paraelectric phase into a low temperature ferroelectric phase of a lower crystal symmetry. The phase transition temperature is usually called the Curie temperature. In most cases, the dielectric constant above the Curie temperature obeys the Curie-Weiss law.

The change of internal energy, $\mathrm{dU}$, of a ferroelectric material subjected to a small strain $d x$, electric displacement $d D_{i}$, and entropy $d S$ can be expressed by

$$
d U=T d S+X_{i j} d x_{i j}+E_{i} d D_{i}
$$

where $\mathrm{T}$ is the temperature of the thermodynamic system. Since most piezoelectric systems are subjected to stress, electric field and temperature variations, it is convenient to express the free energy into the form of the Gibbs energy

$$
d G=-S d T-x_{i j} d X_{i j}-D_{i} d E_{i}
$$

According to the Taylor expansion around a certain equilibrium state, $G_{0}(T)$, the Gibbs free energy can be expanded in terms of the independent variables $T, X$ and $D$

$$
\begin{aligned}
& G=G_{0}(T)+\left(\frac{\partial G}{\partial T}\right) \Delta T+\left(\frac{\partial G}{\partial X_{i j}}\right) X_{i j}+\left(\frac{\partial G}{\partial D_{i}}\right) D_{i}+\frac{1}{2}\left(\frac{\partial^{2} G}{\partial T^{2}}\right) \Delta T^{2} \\
& +\frac{1}{2}\left(\frac{\partial^{2} G}{\partial X_{i j} \partial X_{k l}}\right) X_{i j} X_{k l}+\frac{1}{2}\left(\frac{\partial^{2} G}{\partial D_{i} \partial D_{j}}\right) D_{i} D_{j}+\frac{1}{2}\left(\frac{\partial^{2} G}{\partial T \partial X_{i j}}\right) \Delta T X_{i j} \\
& +\frac{1}{2}\left(\frac{\partial^{2} G}{\partial T \partial D_{j}}\right) \Delta T D_{j}+\frac{1}{2}\left(\frac{\partial^{2} G}{\partial X_{i i} \partial D_{k}}\right) X_{i j} D_{k}+\cdots \cdots
\end{aligned}
$$

This phenomenological theory treats the material in question as a continuum without regard to local microstructure variations [8]. Although the treatment itself does not provide physical insight on the origin of ferroelectricity, it has been demonstrated as the most powerful tool for the explanation of some ferroelectric phenomena such as Curie-Weiss relation, the order of phase transition and abnormal electromechanical behaviors [9]. Equation (3) can be rewritten as [10]: 


$$
\begin{aligned}
& \Delta G=a_{1}\left(P_{1}^{2}+P_{2}^{2}+P_{3}^{2}\right)+a_{11}\left(P_{1}^{4}+P_{2}^{4}+P_{3}^{4}\right)+a_{12}\left(P_{1}^{2} P_{2}^{2}+P_{2}^{2} P_{3}^{3}+P_{1}^{2} P_{3}^{2}\right) \\
& +a_{111}\left(P_{1}^{6}+P_{2}^{6}+P_{3}^{6}\right)+a_{112}\left[P_{1}^{4}\left(P_{2}^{2}+P_{3}^{2}\right)+P_{2}^{4}\left(P_{1}^{2}+P_{3}^{2}\right)+P_{3}^{4}\left(P_{2}^{2}+P_{1}^{2}\right)\right] \\
& +a_{123} P_{1}^{2} P_{2}^{2} P_{3}^{2}-\frac{1}{2} s_{11}\left(X_{1}^{2}+X_{2}^{2}+X_{3}^{2}\right)-s_{12}\left(X_{1} X_{2}+X_{2} X_{3}+X_{1} X_{3}\right) \\
& -\frac{1}{2} s_{44}\left(X_{4}^{2}+X_{5}^{2}+X_{6}^{2}\right)-Q_{11}\left(X_{1} P_{1}^{2}+X_{2} P_{2}^{2}+X_{3} P_{3}^{2}\right) \\
& -Q_{12}\left[X_{1}\left(P_{2}^{2}+P_{3}^{2}\right)+X_{2}\left(P_{1}^{2}+P_{3}^{2}\right)+X_{3}\left(P_{2}^{2}+P_{1}^{2}\right)\right] \\
& -Q_{44}\left(X_{4} P_{2} P_{3}+X_{4} P_{2} P_{3}+X_{6} P_{2} P_{1}\right)
\end{aligned}
$$

where the coefficients, $a_{1}, a_{2}$, and $a_{3}$ can be identified from equation (4) and $\mathrm{s}$ and $Q$ are known as the elastic compliance and the electrostrictive coefficient, respectively.

For a ferroelectric perovskite, equation (4) can be further simplified if the crystal structure and the corresponding polarization are taken into consideration. The polarization for cubic, tetragonal, orthorhombic and rhombohedral ferroelectrics is listed in Table 1, where 1, 2, and 3 denotes the a-, b-, and c- axis in a unit cell.

\section{Cubic}

Tetragonal

Orthorhombic

Rhombohedral

$$
\begin{gathered}
P_{1}^{2}=P_{2}^{2}=P_{3}^{2}=0 \\
P_{1}^{2}=P_{2}^{2}=0, P_{3}^{2} \neq 0 \\
P_{1}^{2}=P_{2}^{2} \neq 0, P_{3}^{2}=0 \\
P_{1}^{2}=P_{2}^{2}=P_{3}^{2} \neq 0
\end{gathered}
$$

Table 1. The polarization for cubic, tetragonal, orthorhombic, and rhombohedral structures.

Thus, considering the tetragonal ferroelectric system in the absence of external electrical field and without temperature change, the electric displacement, $\mathrm{D}$, equals to the polarization in the direction parallel to the c- axis. The free energy can then be further simplified as

$$
G=G_{0}(T)+\frac{1}{2} a_{1} P^{2}+\frac{1}{4} a_{2} P^{4}+\frac{1}{6} a_{3} P^{6}+\frac{1}{2} s X^{2}+Q X P^{2}+\cdots \cdots
$$

where $a_{1}=\beta$ (T - T $T_{c}$ ) with $\beta$ a positive constant, $T_{c}$ is the Curie temperature for second-order phase transitions or the Curie-Weiss temperature ( $\neq$ the Curie temperature) for first-order phase transition.

\section{Point defects}

Point defects occur in crystal lattice where an atom is missing or replaced by an foreign atom. Point defects include vacancies, self-interstitial atoms, impurity atoms, substitutional atoms. It has been long realized even the concentration of point defects in solid is considered to be very low, they can still have dramatic influence on materials properties [11,12]:

- Vacancies and interstitial atoms will alternate the transportation of electrons and atoms within the lattice.

- Point defects create defect levels within the band gap, resulting in different optical properties. Typical examples include $\mathrm{F}$ centers in ionic crystals such as $\mathrm{NaCl}$ and $\mathrm{CaF}_{2}$. Crystals with $\mathrm{F}$ centers may exhibit different colors due to enhanced absorption at visible range (400 - $700 \mathrm{~nm}$ ). 
The most important point defect in ferroelectric perovskites is oxygen vacancies. Perovskiterelated structures exhibit a large diversity in properties ranging from insulating to metallic to superconductivity, magneto-resistivity, ferroelectricity, and ionic conductivity. Owing to this wide range of properties, these oxides are used in a great variety of applications. For example, $(\mathrm{Ba}, \mathrm{Sr}) \mathrm{TiO}_{3}$ and $\mathrm{Pb}(\mathrm{Zr}, \mathrm{Ti}) \mathrm{O}_{3}$ are high-dielectric constant materials being considered for dynamic and nonvolatile random access memories, $\mathrm{Pb}(\mathrm{Zr}, \mathrm{Ti}) \mathrm{O}_{3}$ is high piezoelectric constant material being used for actuators and transducers, and $\mathrm{LaMnO}_{3}$ and $(\mathrm{La}, \mathrm{Sr}) \mathrm{CoO}_{3}$ are being used as electrode materials in solid oxide fuel cells. Oxygen vacancies in perovskites are particularly of interests due partly to the loosely packed oxygen octahedra that lead to high mobility of oxygen vacancies. In perovskite ferroelectrics, a lot of works have been conducted to understand the behaviors of oxygen vacancies under the influence of external fields, such as electrical, stress and thermal fields, sometimes as a function of temperatures [13]. Oxygen vacancies play an essential role on ferroelectric fatigue during the operation of a ferroelectric component subjected to continuous load of electrical or stress fields, though many other factors such as microcracks [14], spatial charges [21], electrodes[15], surfaces and interfaces[16], voids, grain boundaries [21] may also lead to ferroelectric fatigue. The accumulation of oxygen vacancies in the electrode/ferroelectric interface has been confirmed by experimental studies. This oxygen deficient interface region could either screen external electrical field [24,17] or pin domain walls [18], both of which will reduce the polarizability of the ferroelectric thin films. Although ferroelectric fatigue induced by the accumulation of oxygen vacancies is considered to be permanent, thermal or UV treatment in oxygen rich environment can sometimes partially recover the switchability. Another option is to use conductive oxide electrode materials such as LSCO or YBCO which can serve as sinks for oxygen vacancies and prevent their accumulation at the electrode/film interface $[19,20]$.

Recently, efforts have been made on hydrothermal synthesis of $\mathrm{BaTiO}_{3}$ nanoparticles of various sizes to understand the ferroelectric size effect by using $\mathrm{BaCl}_{2}$ and $\mathrm{TiO}_{2}$ as the starting materials. [21,22]. The growth of $\mathrm{BaTiO}_{3}$ nanoparticles is commonly believed to follow a two step reaction mechanism: 1) the formation of Ti-O matrix, 2) the diffusive incorporation of $\mathrm{Ba}^{2+}$ cations. The second step is believed to the rate determinant process. Due to the presence of $\mathrm{H}_{2} \mathrm{O}, \mathrm{OH}$ - groups are always present in hydrothermal $\mathrm{BaTiO}_{3}$. As a result, some studies have been performed to understand $\mathrm{OH}$ - effects on ferroelectricity. D. Hennings et al reported that a reduction of hydroxyl groups in $\mathrm{BaTiO}_{3}$ nanoparticles promotes cubic-to-tetragonal phase transition [23]. Similar results had also been obtained by other studies on $\mathrm{BaTiO}_{3}$ particles with sizes varying from $20 \mathrm{~nm}$ to $100 \mathrm{~nm}$ [24,25]. These experimental observations imply that point defects and possibly the associated electrical fields can lead to structural phase transition, as suggested by the soft-mode theory.

Currently, point defects in ferroelectrics are mostly studied by optical methods such as FTIR spectroscopy or Raman spectroscopy. For $\mathrm{BaTiO}_{3}$, the stretching vibration of lattice $\mathrm{OH}-$ groups occurs at $3462.5-3509.5 \mathrm{~cm}^{-1}$, characterized by a sharp absorption peak [26]. In contrast, surface $\mathrm{OH}$ - groups are characterized by a broad absorption peak located at 3000 $3600 \mathrm{~cm}^{-1}[44,27]$ due to the uncertain chemical environment on surface region. Raman spectroscopy is also a powerful tool to understand the size effect of ferroelectrics, which is quite sensitive to local variation of lattice structure. S. Wada et al. reported that $\mathrm{OH}$ - groups in $\mathrm{BaTiO}_{3}$ correspond to an $810 \mathrm{~cm}^{-1}$ Raman shift [28]. As point defects can create extra 
electron levels in the band gap, photoluminescent spectroscopy had also been utilized to study the band structure of $\mathrm{BaTiO}_{3}$, which is frequently conducted at low temperatures. Some other techniques such as HRTEM [29] and AFM [30] have also been used to study point defects.

\section{Dislocations in ferroelectrics}

The LGD theory predicts that dislocations in a ferroelectric will change the local ferroelectric behaviors around them. Considering a perovskite ferroelectric single domain with a tetragonal structure, the coordinate system is defined as $x / /[100], y / /[010]$, and $z / /[001]$ with the spontaneous polarization, $P_{3}$, parallel to the $\mathrm{z}$ axis and $P_{1}=P_{2}=0$. The variation of piezoelectric coefficients induced by a $\{100\}$ edge dislocation can be found with a method derived from combination of the Landau-Devonshire free energy equation [10] and dislocation theory [31]. As previous works suggest [32], the elastic Gibbs free energy around an edge dislocation can be modified as

$$
\begin{aligned}
& G\left[P, T, \sigma_{i j}(x, y)\right]=G_{0}+a_{1}^{*} P^{2}+a_{11} P^{4}+a_{111} P^{6}+\frac{1}{2} s_{11}\left(\sigma_{11}^{2}+\sigma_{22}^{2}\right. \\
& \left.\sigma_{33}^{2}\right)+s_{12}\left(\sigma_{11} \sigma_{22}+\sigma_{11} \sigma_{33}+\sigma_{22} \sigma_{33}\right)+\frac{1}{2} s_{44} \sigma_{12}^{2}+E_{\text {core }}
\end{aligned}
$$

with

$$
a_{1}^{*}\left[T, \sigma_{i j}(x, y)\right]=a_{1}-\left[Q_{11} \sigma_{33}+Q_{12}\left(\sigma_{11}+\sigma_{22}\right)\right]
$$

where $G_{0}$ is the free energy in the paraelectric state, $a_{1}, a_{11}$ and $a_{111}$ are the dielectric stiffness constants at constant stress, $\sigma_{i j}$ is the internal stress field generated by an edge dislocation, $P$ is the spontaneous polarization parallel to the polar axis, $s_{i j}$ is the elastic compliance at constant polarization, $E_{\text {core }}$ is the dislocation core energy and $Q_{i j}$ represents the electrostriction coefficients. The stress field generated by an edge dislocation is well documented in the literature and is known as

$$
\begin{gathered}
\sigma_{11}=-\frac{\mu b}{2 \pi(1-v)} \frac{y\left(3 x^{2}+y^{2}\right)}{\left(x^{2}+y^{2}\right)^{2}}, \sigma_{22}=\frac{\mu b}{2 \pi(1-v)} \frac{y\left(x^{2}-y^{2}\right)}{\left(x^{2}+y^{2}\right)^{2}} \\
\sigma_{33}=v\left(\sigma_{11}+\sigma_{22}\right), \sigma_{12}=\frac{\mu b}{2 \pi(1-v)} \frac{x\left(x^{2}-y^{2}\right)}{\left(x^{2}+y^{2}\right)^{2}} \\
\sigma_{13}=\sigma_{23}=0
\end{gathered}
$$

where $\mu$ is the shear modulus, $b$ is the Burgers vector and $v$ is Poisson's ratio. A schematic plot of the stress field surrounding an edge dislocation is given in Fig. 1a.

The variation of the spontaneous polarization associated with the stress field due to an edge dislocation is then found by minimizing the modified Landau-Devonshire equation with respect to polarization $[(\partial G / \partial P)=0]$. Upon rearrangement, this gives [7] 


$$
P^{2}\left[T, \sigma_{i j}(x, y)\right]=\frac{-a_{11}+\sqrt{\left(a_{11}^{2}-3 a_{1}^{*}\left[T, \sigma_{i j}(x, y)\right] a_{111}\right)}}{3 a_{111}}
$$

Once the polarization is known for a given position, the piezoelectric coefficient, $d_{33}$, can be calculated by using [2]

$$
d_{33}=2 \varepsilon_{33} Q_{11} P
$$

where $d_{33}$ is the piezoelectric coefficient along the polar axis.

\begin{tabular}{|c|c|c|c|}
\hline \multicolumn{2}{|c|}{ Elastic Constants } & \multicolumn{2}{c|}{ Piezoelectric Coefficients } \\
\hline$C_{11}(\mathrm{GPa})$ & 275 & $\mathrm{~T}(\mathrm{~K})$ & 298 \\
\hline$C_{12}(\mathrm{GPa})$ & 179 & $a_{1}\left(V m \mathrm{C}^{-1}\right)$ & $3.34 \times 10^{5}(T-381)$ \\
\hline$C_{13}(\mathrm{GPa})$ & 152 & $a_{11}\left(V m^{5} \mathrm{C}^{-3}\right)$ & $4.69 \times 10^{6}(T-393)-2.02 \times 10^{8}$ \\
\hline$C_{33}(\mathrm{GPa})$ & 165 & $a_{111}\left(V m^{9} \mathrm{C}^{-5}\right)$ & $-5.52 \times 10^{7}(T-393)+2.76 \times 10^{9}$ \\
\hline$C_{44}(\mathrm{GPa})$ & 54 & $Q_{11}\left(m^{4} C^{-2}\right)$ & 0.11 \\
\hline$C_{66}(\mathrm{GPa})$ & 113 & $Q_{12}\left(m^{4} C^{-2}\right)$ & -0.045 \\
\hline
\end{tabular}

Table 2. Elastic and piezoelectric properties required for theoretical calculations for barium titanate single crystals.

The elastic compliance, dielectric stiffness constants and electrostriction coefficients used in the calculation were found for $\mathrm{BaTiO}_{3}$ from other works [33,34]. The resulting $\mathrm{d}_{33}$ contour around the dislocation core is plotted and shown in Fig. 1b, where some singular points resulted from the infinite stress at the dislocation core are discarded. It is clearly seen that the piezoelectric coefficient $d_{33}$ deviate from the standard value $(86.2 \mathrm{pm} / \mathrm{V}$ at $293 \mathrm{~K})$, due to the presence of the stress field. The area dominated by transverse compressive stresses exhibits an enhanced piezoelectric response while the area dominated by tensile stresses shows reduced effects. Note that the influence of stress field shows asymmetric effects on the piezoelectric coefficients due to the combination of equations (7) and (9). This simple calculation also suggests that the area significantly influenced by an edge dislocation could easily reach tens of nanometers as a result of the dislocation long-range stress field. In addition, dislocation stress field will also change the local properties of its surrounding area, like chemical reactivity, electron band structure, absorption of molecules and so on. However, stress field solely sometimes is not sufficient to describe all effects; a fully understanding of dislocation effects on ferroelectricity requires in-depth knowledge on electrical fields induced by the charged core area, which is currently not fully addressed in literature. 


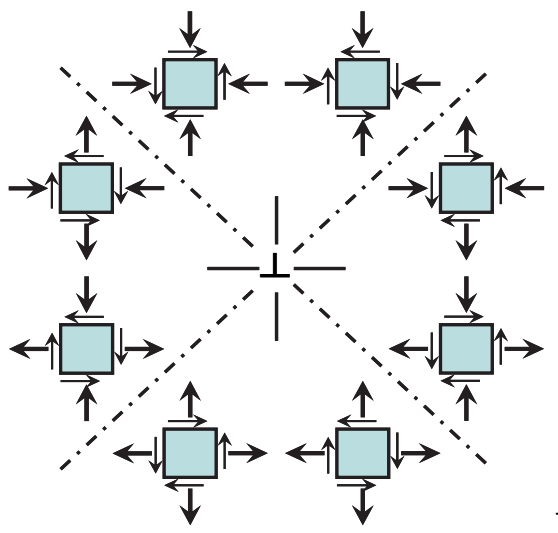

(a)

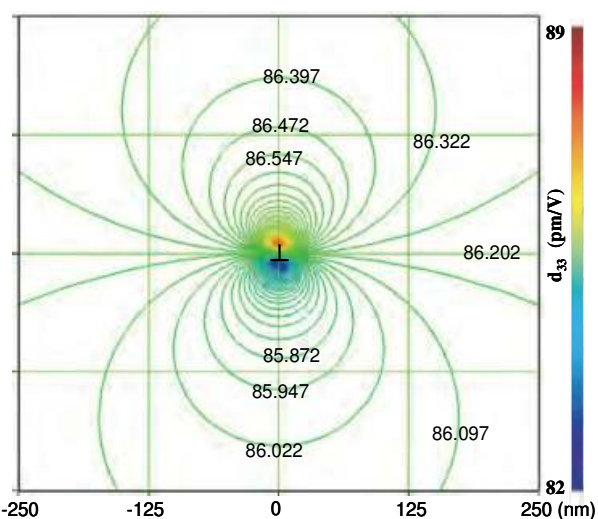

(b)

Fig. 1. The schematic representation of the stress field around an edge dislocation (a) and the resulting piezoelectric coefficient contour (b) calculated from the Landau-Devenshire theory.

Recently, many studies have been performed to understand dislocation effects on ferroelectricity. M. W. Chu et al. [35] found that misfit dislocations between PZT islands and $\mathrm{SrTiO}_{3}$ substrate (height: $4 \mathrm{~nm}$, width: $8 \mathrm{~nm}$ ) can lead to polarization instability, as confirmed by HRTEM and PFM tests. C. L. Jia et al [36] found that the elastic stress field of a dislocation in $\mathrm{SrTiO}_{3} / \mathrm{PZT} / \mathrm{SrTiO}_{3}$ multilayered structures, even if it is located in regions far from the ferroelectric material, can have a determinant effect on ferroelectricity. A decrease of local spontaneous polarization of $48 \%$ was obtained by calculation. C. M. Landis et al. [37] found by non-linear finite element method (FEM) simulation that the stress field of dislocations can pin domain wall motions. L. Q. Chen et al [38] found by phase field simulations that misfit dislocations will alternate ferroelectric hysteresis. D. Liu et al performed nano indentation tests on individual $90^{\circ}$ and $180^{\circ}$ domains on $\mathrm{BaTiO}_{3}$ single crystal and found that in an area free of dislocations the nucleation of dislocations induced by an indenter with tip radius of several tens of nanometers will be accompanied by the formation of ferroelectric domains of complex domain patterns, as confirmed by PFM tests. Recently, dislocation effects had been extended to other areas. For example, a theoretical work even predicted that dislocations may induce multiferroic behaviors in ordinary ferroelectrics [39]. In a recent study, the Author's group found that there exists a critical size below which dislocations in barium titanate $\left(\mathrm{BaTiO}_{3}\right)$, a model ferroelectric, nanocubes can not exist. While studying the etching behaviors of $\mathrm{BaTiO}_{3}$ nanocubes with a narrow size distribution by hydrothermal method, it was confirmed that the etching behaviors of $\mathrm{BaTiO}_{3}$ nanocubes are size dependent; that is, larger nanocubes are more likely to be etched with nanosized cavities formed on their habit facets. In contrast, smaller nanocubes undergo the conventional Ostwald dissolution process. A dislocation assisted etching mechanism is proposed to account for this interesting observation. This finding is in agreement with the classical description of dislocations in nanoscale, as described theoretically [40].

\section{Dislocation size effect}

The author's group reported an interesting observation on BaTiO3 nanocubes synthesized through a modified hydrothermal method. Detailed analysis is provided as follows. The 
experimental procedure is relatively simple. First a small amount of $\mathrm{NaOH}: \mathrm{KOH}$ mixture was placed into a Teflon-lined autoclave. After the addition of $\mathrm{BaCl}_{2}$ and $\mathrm{TiO}_{2}$ (anatase), the autoclave was sealed and heated at $200^{\circ} \mathrm{C}$ for 48 hours. After reaction, the product was collected by filtering and washing thoroughly with deionized water and diluted $\mathrm{HCl}$ acid. The reaction is as follows:

$$
2 \mathrm{NaOH}+\mathrm{TiO}_{2}+\mathrm{BaCl}_{2} \rightarrow \mathrm{BaTiO}_{3}+2 \mathrm{NaCl}+\mathrm{H}_{2} \mathrm{O}
$$

The free Gibbs energy of the formation of $\mathrm{BaTiO}_{3}$ at $200^{\circ} \mathrm{C}$ was calculated. The enthalpy of formation is

$$
\begin{aligned}
\Delta \mathrm{H} & =2 \Delta \mathrm{H}_{\mathrm{NaCl}}+\Delta \mathrm{H}_{\mathrm{H} 2 \mathrm{O}}+\Delta \mathrm{H}_{\mathrm{BaTiO}^{-}}\left(2 \Delta \mathrm{H}_{\mathrm{NaOH}}+\Delta \mathrm{H}_{\mathrm{BaCl} 2}+\Delta \mathrm{H}_{\mathrm{TiO} 2}\right) \\
& =-2 \times 411.2-285.830-1659.8-(-2 \times 425.6-855.0-944.0)=-117.83 \mathrm{KJ} \cdot \mathrm{mol}^{-1}
\end{aligned}
$$

The entropy of formation is

$$
\begin{aligned}
\Delta S & =2 \mathrm{~S}_{\mathrm{NaCl}}+\mathrm{S}_{\mathrm{H} 2 \mathrm{O}}+\mathrm{S}_{\mathrm{BaTiO} 3}-\left(2 \mathrm{~S}_{\mathrm{NaOH}}+\Delta \mathrm{S}_{\mathrm{BaCl} 2}+\mathrm{S}_{\mathrm{TiO} 2}\right) \\
& =2 \times 72.1+69.95+108.0-(2 \times 64.4+123.67+50.62)=19.06 \mathrm{JoC} \cdot \mathrm{mol}^{-1}
\end{aligned}
$$

Then the free Gibbs energy of formation at reaction temperature $200^{\circ} \mathrm{C}$ is

$$
\begin{aligned}
\Delta \mathrm{G} & =\Delta \mathrm{H}-\mathrm{T} \Delta \mathrm{S} \\
& =-117.83-19.06 \times 473 / 1000=-126.845 \mathrm{KJ}^{\mathrm{mol}}{ }^{-1}
\end{aligned}
$$

It can be seen that the formation of $\mathrm{BaTiO}_{3}$ proceed easily at $200{ }^{\circ} \mathrm{C}$. Our experiments had shown that $\mathrm{BaTiO}_{3}$ nanocubes can be formed at temperatures as low as $180^{\circ} \mathrm{C}$, as shown in Fig. 2, much lower than the temperature required by conventional solid-state reactions. All the diffraction peaks can be indexed to tetragonal $\mathrm{BaTiO}_{3}(\mathrm{P} 4 \mathrm{~mm}, \mathrm{JCPD}$ 81-2203).

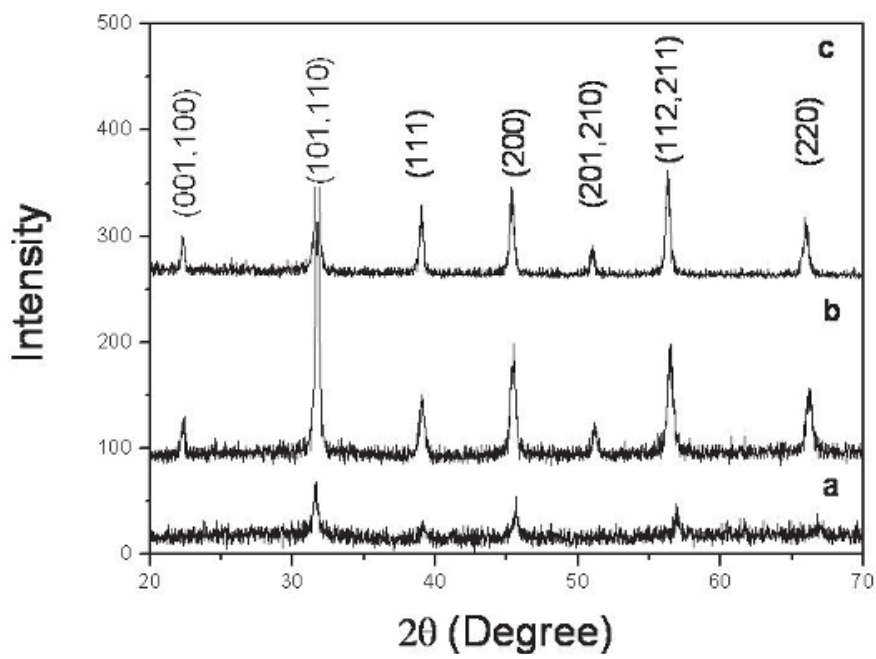

Fig. 2. XRD patterns of $\mathrm{BaTiO}_{3}$ nanocubes synthesized at a) $180^{\circ} \mathrm{C}$, b) $200^{\circ} \mathrm{C}$ and c) $220^{\circ} \mathrm{C}$. 
After the synthesis of $\mathrm{BaTiO}_{3}$ nanocubes, we also studied their etching behaviors in hydrothermal environment. The etching process of $\mathrm{BaTiO}_{3}$ nanocubes was carried out in diluted $\mathrm{HCl}$ solution $(1 \mathrm{M})$. The $\mathrm{BaTiO}_{3}$ nanocubes were first mixed with $\mathrm{HCl}$ solution and then the mixture was treated in hydrothermal environment at $120^{\circ} \mathrm{C}$ for 2.5 hours. The reaction time and temperature had been optimized in consideration that over reaction may lead to the formation of $\mathrm{TiO}_{2}$, as shown in Fig. 3 and Fig. 4.

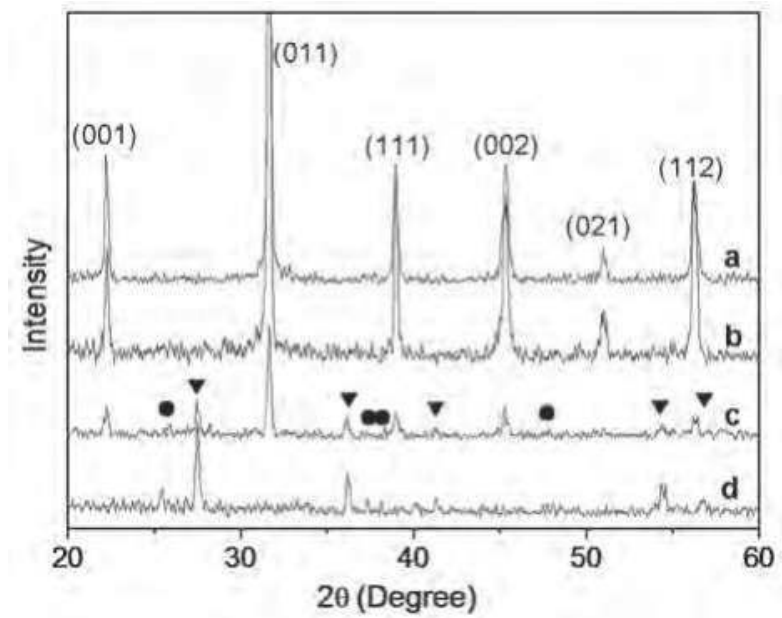

Fig. 3. XRD patterns of the final products after hydrothermal treatment at $120^{\circ} \mathrm{C}$ for various time: a) $30 \mathrm{~min}$, b) $40 \mathrm{~min}$, c) $50 \mathrm{~min}$, d) $60 \mathrm{~min}$. The $\boldsymbol{\nabla}$ and $\bullet$ marks correspond to rutile and anatase $\mathrm{TiO}_{2}$, respecitively.

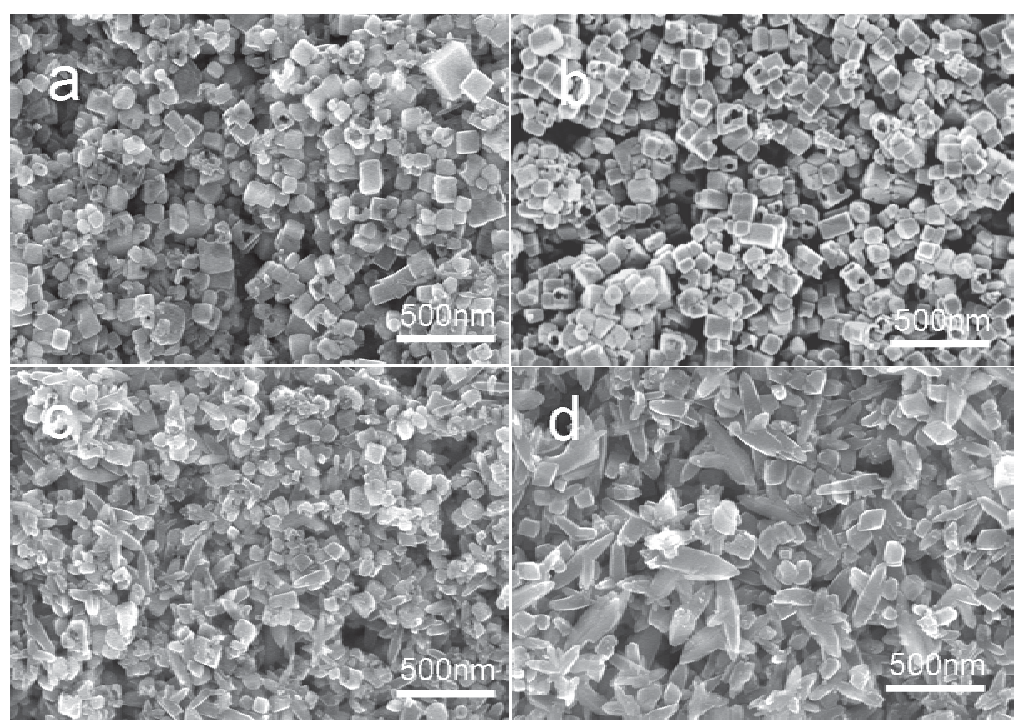

Fig. 4. SEM images of the final products after hydrothermal treatment at $120^{\circ} \mathrm{C}$ for a) $30 \mathrm{~min}$, b) $40 \mathrm{~min}$, c) $50 \mathrm{~min}$, and d) $60 \mathrm{~min}$. 
Fig. 5a shows a typical SEM image obtained on the as-synthesized product. It can be seen that all nanoparticles exhibit a cubic morphology with sizes of $\sim 30-100 \mathrm{~nm}$. FTIR analysis reveals that the $\mathrm{BaTiO}_{3}$ nanocubes contain a very small amount of lattice $\mathrm{OH}$ - groups, considerably less than $\mathrm{BaTiO}_{3}$ nanoparticles synthesized by regular hydrothermal method. Fig. $5 \mathrm{~b}$ shows a typical SEM image of the etched product, which reveals particle sizes smaller than that of the as-synthesized product (Fig. 5a). Besides, it is also interesting to note the fact that small cavities are formed on some nanocubes.

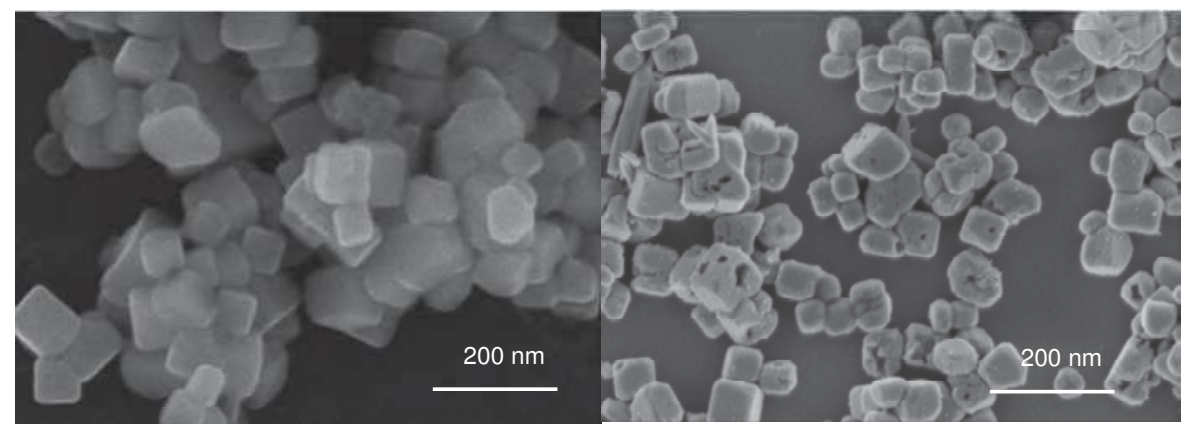

(a)

(b)

Fig. 5. SEM image of BaTiO3 nanocubes before (a) and after (b) hydrothermal etching. (Copyright 2008 @ American Chemical Society.)

A statistical analysis reveals that these cavities only present on nanocubes greater than $\sim 60$ nm. Fig. 5 shows SEM images of nanocubes of different sizes obtained under the same experimental conditions. It can be clearly seen that nanocubes smaller than $\sim 60 \mathrm{~nm}$ remain intact, while cavities are selectively formed on those greater than $\sim 60 \mathrm{~nm}$. The etching process was initiated on the surface and can penetrate all the way through a nanocube. In most case, there is only one etch pit in one nanocube while occasionally there are two or three etch pits observed.
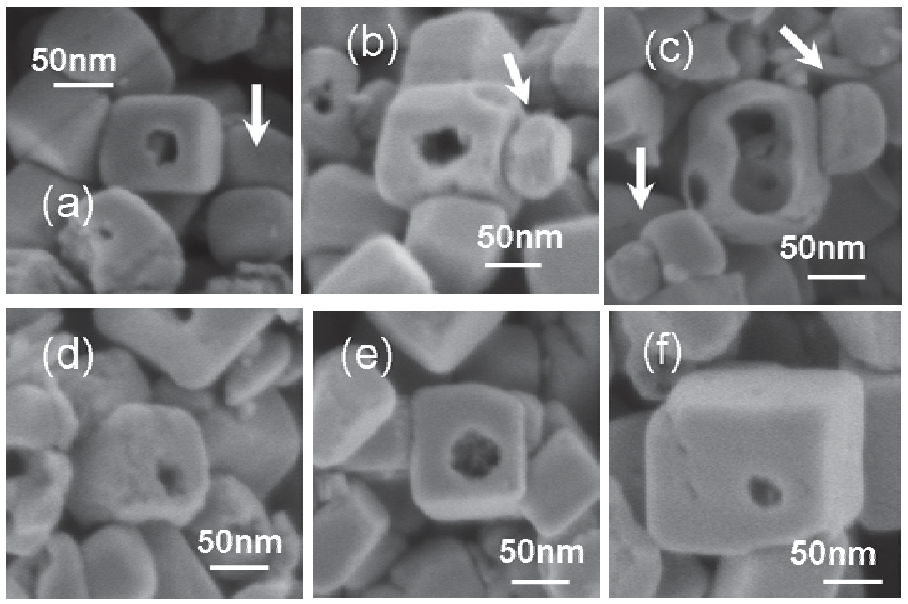

Fig. 6. SEM images of $\mathrm{BaTiO}_{3}$ nanocubes after hydrothermal etching. 
All the observation seems to be in controversy to the Ostwald dissolution mechanism, which predicts that small particles will dissolve first during a chemical reaction. However, our experiments reveal that smaller $\mathrm{BaTiO}_{3}$ nanocubes show a better chance to remain intact though their corners and edges seem to have dissolved. The dissolution of corners and edges could be understood based on the Gibbs-Thompson relation. The Gibbs-Thompson relation suggests that, for a small particle, its corners and edges have enhanced chemical reactivity and their dissolutions are energetically favored. The Gibbs-Thompson relation also implies that smaller nanocubes have higher dissolubility and should dissolve first in compensation of the growth of larger ones.

Fig. 7a shows a typical HRTEM image taken on a $\mathrm{BaTiO}_{3}$ nanocube with length of $\sim 15 \mathrm{~nm}$. It is evident that the nanocube is enclosed by (100) and (110) habit facets due to their high chemical stabilities [41]. Fig. $7 \mathrm{~b}$ shows the fast Fourier transformation (FFT) image of Fig. $7 \mathrm{a}$, which shows that the nanocube contains cubic lattices with lattice parameters of $\sim 0.4$ $\mathrm{nm}$, suggesting that the nanocube is in cubic non-ferroelectric phase, in agreement with many previous studies. A careful examination of the lattice on the enlarged FFT filter image (Figure 7c) shows that the nanocube exhibit perfect lattice without dislocation or stacking faults. However, on the surface region, defective layers with distinct structures were formed due possibly to the presence of non-stoichiometric Ti-O layer as a result of $\mathrm{Ba}^{2+}$ dissolution in acid $[42,43]$. As suggested by previous studies, the formation of $\mathrm{BaTiO}_{3}$ in base contains two steps, namely the precipitation of Ti-O networks and the incorporation of $\mathrm{Ba}^{2+}$. Similarly, the dissolution of $\mathrm{BaTiO}_{3}$ in acid contains outward diffusion of $\mathrm{Ba}^{2+}$ followed by phase transition of Ti-O network into $\mathrm{TiO}_{2}$. As the Ti-O surface layers prevent $\mathrm{Ba}^{2+}$ from dissolution out of the Ti-O matrix, it can be expected that the dissolution rate of $\mathrm{BaTiO}_{3}$ will be slowed down as the reaction proceeds. It is also possible that at certain stage of the reaction the particles may contain a $\mathrm{BaTiO}_{3}$ core surrounded by a $\mathrm{TiO}_{2}$ shell.

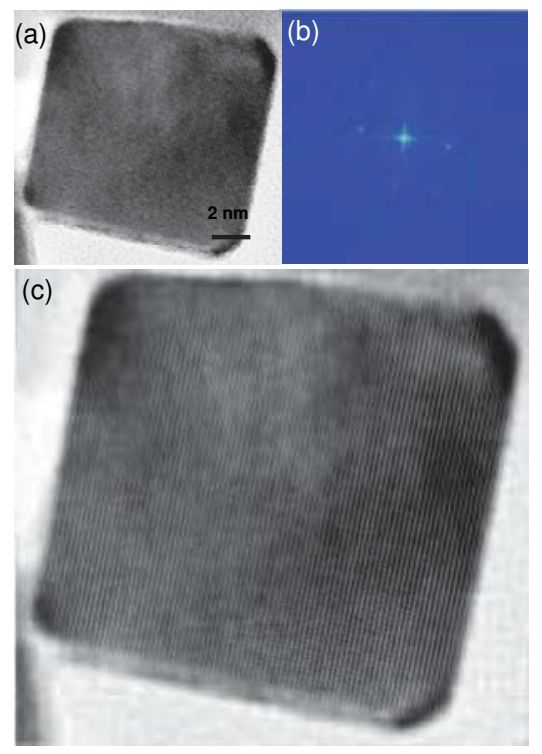

Fig. 7. HRTEM image taken on a BaTiO3 nanocube (a), the corresponding FFT pattern (b), and filtered image (c). 
In contrast, the existence of dislocation inside a nanoparticle will dramatically change the way of the dissolution of nanoparticles. As dislocated regions are highly strained, regions with dislocations usually exhibit enhanced chemical reactivity. Preferential removal of atoms in the dislocation core area has been extensively observed on various materials such as metals, semiconductors and insulators. Although point defects such as the aforementioned oxygen vacancies and hydroxyl groups may also increase local etching rate, unlike extended defects, their effect is limited in a very small region and, even if there is any, should be observable on all nanocubes of various sizes no matter they are greater or smaller than $60 \mathrm{~nm}$.

This observation also implies that there exists a critical size for dislocation to present inside $\mathrm{BaTiO}_{3}$ nanocubes, and possibly all other nanoparticles. To understand this, we need to look into more details about the elastic theory of dislocation in nanoparticles. A literature review reveals that the classical elastic theory indeed predicts a characteristic length below which dislocation can not exist within an isolated nanoparticle [44, 45]. It was suggested that dislocations would be driven out of the crystal spontaneously when the size of the crystal is less than a characteristic length given by $[46,47]$

$$
A_{c} \cong 2 G b / \sigma_{p}
$$

where $G$ is the shear modulus, $b$ is the Burgers vector of the dislocation, and $\sigma_{p}$ is the Peierls stress given by [48]

$$
\sigma_{p}=\frac{3 \sqrt{3}}{2} \frac{a(1-v)}{G b} \tau_{\max }^{2}
$$

where $G$ is the shear modulus of the material, $a$ the lattice parameter, $v$ the Poisson's ratio, and $\tau_{\max }$ the ideal shear strength.

For $\mathrm{BaTiO}_{3}$, the average shear modulus is estimated to be $55 \mathrm{GPa}$ with a method introduced by Watt and Peselnick [49], Burgers vector $b=a[110] / 2=0.28 \mathrm{~nm}$, and the ideal shear strength of $5.5 \mathrm{GPa}$, as determined by nanoindentation test [50]. Bu substituting the data into equation (13), $A_{c}$ for spherical $\mathrm{BaTiO}_{3}$ nanoparticles is estimated to be $\sim 22 \mathrm{~nm}$. The calculated value is smaller than that determined experimentally due to a combination of the following factors: (1) the assumption of spherical shape used in the original model may not be fully transferrable to cubic shaped nanoparticles; (2) the elastic anisotropy of $\mathrm{BaTiO}_{3}$ means that an average shear modulus may not be sufficiently accurate; (3) the presence of the Ti-O surface layers may also lead to alternate the case from the model; (4) possibly the most important, ferroelectric size effects could also play a role. In fact, all these possibilities lie on the fact that the elastic properties of $\mathrm{BaTiO}_{3}$ nanocubes could deviate from the bulk values. As a result, we performed first principle ab-initio calculation on $\mathrm{BaTiO}_{3}$ with the CASTEP module of Materials Studio in the assumption of the nanocubes having a cubic lattice structure. The calculated elastic modulus are $\mathrm{C}_{11}=284.9 \mathrm{GPa}, \mathrm{C}_{12}=110.8 \mathrm{GPa}, \mathrm{C}_{44}$ (shear modulus, $G)=116.2 \mathrm{GPa}$. The computed $\mathrm{C}_{12}$ and $\mathrm{C}_{44}$ agree well with experimental values, while $C_{11}$ is $\sim 10 \%$ greater than the experimental value [51]. Inserting $C_{44}$ to Equation (13) yields a characteristic length of $46.5 \mathrm{~nm}$, which is much closer to the observed critical length. This calculation suggests that ferroelectric size effect has to be considered while describing the etching behaviors of $\mathrm{BaTiO}_{3}$ nanocubes. As discussed above, this critical size 
effect is expected to be observed in other nanostructured materials. This had recently been demonstrated in gallium nitride $(\mathrm{GaN})[52]$.

\section{6. (111) twins in $\mathrm{BaTiO}_{3}$}

The origin of ferroelectricity can be attributed to extrinsic contribution associated with ferroelectric domain wall and intrinsic contribution from lattice distortion [10]. The extrinsic contributions to ferroelectric properties are dominated by: (a) the population of domains, and (b) the mobility of domain walls. In real ferroelectric materials, additional considerations arise owing to the presence of the crystal surfaces and imperfections. In a perfect crystal without imperfections or space charges, $\rho$ is equal to zero. However, the free charge density is different from the perfect crystal at the surface region or in the neighborhood of defects, which alternatively results in the formation of a charge layer. This charge layer may introduce a depolarization field in the nearby regions. When a ferroelectric crystal is cooled from a paraelectric phase to a ferroelectric phase in the absence of applied fields, different crystal regions may take one of these polarization directions such that the total depolarization energy can be minimized. Each volume of uniform polarization is referred to as a ferroelectric domain, and is bounded by domain walls are referred to as domain walls.

There are two types of domain boundaries for a tetragonal perovskite, the polar axes of which are perpendicular or antiparallel with respect to each other. The walls which separate domains with oppositely orientated polarization are defined as $180^{\circ}$ domain walls and those which separate domains with perpendicular polarization are called $90^{\circ}$ domain walls. Unlike its ferromagnetic counterpart, a perovskite ferroelectric possesses a domain wall width in the order of a few unit cells. Since the length of c- axis of a perovskite tetragonal structure, $c_{T}$, is slightly different from that of the a- axis, $a_{T}$, the polarization vectors on each side of a $90^{\circ}$ domain wall form an angle slightly smaller than $90^{\circ}$. The angle can be calculated by

$$
\alpha=2 \times \tan ^{-1}\left(c_{T} / a_{T}\right)
$$

For $\mathrm{BaTiO}_{3}$, taking $c_{T}=4.04 \AA$ and $a_{T}=3.99 \AA$, one obtains 90.7 o, as illustrated in Fig. 8 .

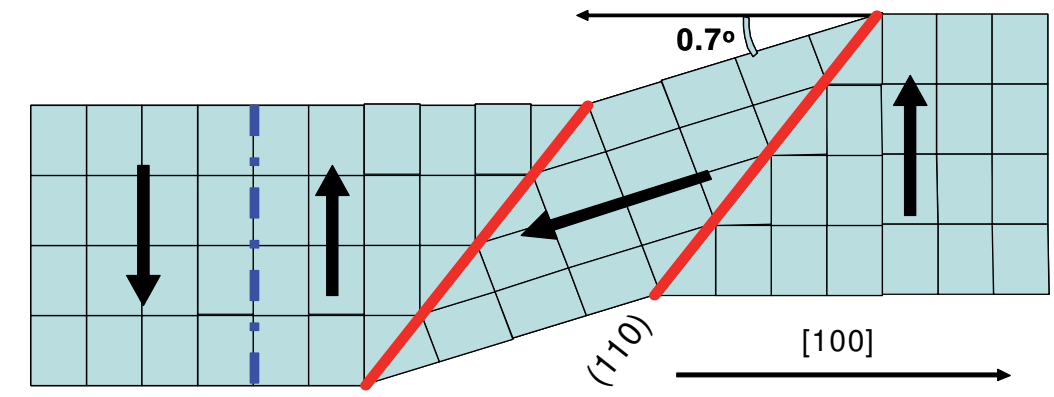

Fig. 8. Schematic illustration of the $180^{\circ}$ and $90^{\circ}$ domain walls in $\mathrm{BaTiO}_{3}$.

Besides regular $90^{\circ}$ and $180^{\circ}$ twin walls, $\mathrm{BaTiO}_{3}$ crystallites containing (111) twins have also been reported. (111) twinned $\mathrm{BaTiO}_{3}$ was first observed in single crystals grown via 
the Remeika method [53] and in bulk ceramics [54] in 1950s. Existing evidences suggest that the formation of (111) twins in ceramics are closely related to the exaggerated growth of the hexagonal $\mathrm{BaTiO}_{3}$ phases on the twin plane which involved oxygen octahedra sharing the face [55]. It has also been suggested that (111) twins can lead to the exaggerated growth of $\mathrm{BaTiO}_{3}$ grains in ceramics following a twin-plane re-entrant edges (TPREs) mechanism [56,57] since the decreasing of activation energy of nucleation on the TPREs.

We recently reported the controlled synthesis of $\mathrm{BaTiO}_{3}$ microcrystallites through a twostep synthesis approach $[58,59]$. The synthesis method is quite similar to the synthesis of BaTiO3 nanocubes, except that the starting anatase $\mathrm{TiO}_{2}$ powders were first treated in autoclave for 5 hours. Then, $\mathrm{BaCl}_{2}$ and water were added into the autoclave, followed by heat treatment at $180^{\circ} \mathrm{C}$ for different period of time up to 20 days. It is found that the pretreated $\mathrm{TiO}_{2}$ is essential for the synthesis of penetrated $\mathrm{BaTiO}_{3}$. The crystallites exhibit penetrated morphologies and contain multiple (111) twins, originated from amorphous $\mathrm{TiO}_{2}$ clusters.
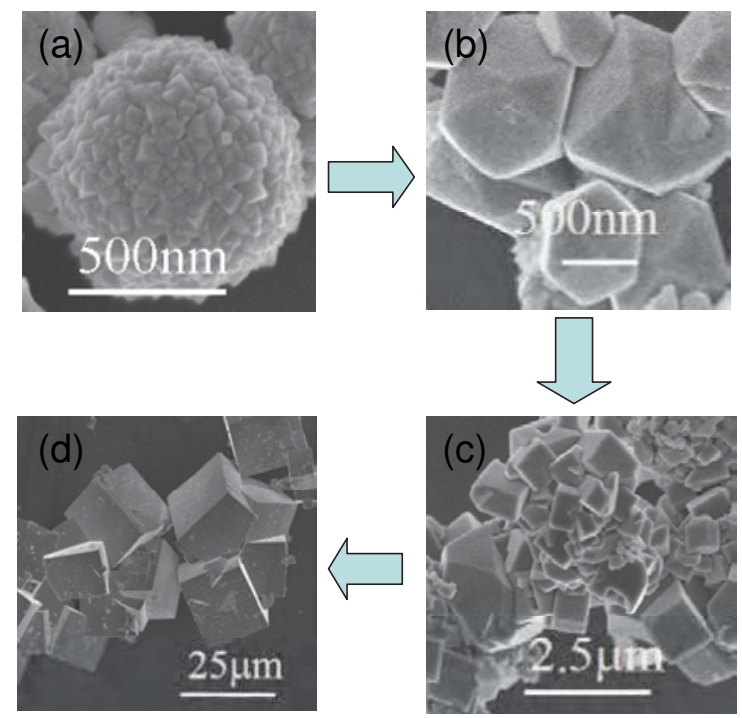

Fig. 9. SEM images of penetrated $\mathrm{BaTiO}_{3}$ microcrystallite obtained at different synthesis stages. (Copyright 2010 @ Royal Society of Chemistry).

Figure 10a shows the photograph of (111) twined $\mathrm{BaTiO}_{3}$ nanoparticles before and after UV irradiation. The UV-vis absorption spectra reveal the presence of defect energy levels after UV irradiation. The color of the powders changes from pale yellow to dark brown after UV irradiation. Oxygen vacancies create additional energy levels within the forbidden energy gap of titanates, usually $0.2-0.3 \mathrm{eV}$ below the conduction band edge [60,61].Figure 10c shows the XPS spectra of Ti- $2 p$ electrons before and after UV irradiation. A careful curve fitting shows that a shoulder peak appears at position $\sim 1.3 \mathrm{eV}$ lower than that of $\mathrm{Ti}^{4+}$ cations, suggesting the presence of $\mathrm{Ti}^{3+}$ cations [62]. The mechanism for the formation of $\mathrm{Ti}^{3+}$ cations is discussed as follows. As the valence band of $\mathrm{BaTiO}_{3}$ is dominated by $\mathrm{O}-2 p$ orbits, whereas the conduction band is the Ti- $3 d$ orbits [17], electrons of O- $2 p$ orbits can be excited by UV 
photons to the Ti- $3 d$ orbits, resulting in the formation of gaseous oxygen and leaving behind oxygen vacancies inside the microcrystallites. The excited electrons are either trapped by $\mathrm{Ti}^{4+}$ to form $\mathrm{Ti}^{3+}$ centers or are trapped by oxygen vacancies to form F-centers, both of which could have strong absorption at visible region, resulting in the observed photochromic effect. However, it is still unclear why the photochromic effect can hardly be observed on regular $\mathrm{BaTiO}_{3}$ nanocubes without (111) twins. It seems that the (111) twin walls may also have a role during the process described above. Previous HRTEM investigations had revealed that the (111) twin walls are composed of $\mathrm{Ba}-\mathrm{O}_{3-\mathrm{x}}-\left[\mathrm{V}_{\mathrm{O}}\right]_{\mathrm{x}}$ instead of $\mathrm{Ba}-\mathrm{O}_{3}$ plane to balance the charge of adjacent $\mathrm{Ti}^{4+}$ ions [63].

Fig. 11 shows the magnetization $(\mathrm{M})$ versus applied magnetic field $(\mathrm{H})$ curves measured at room temperature before and after the UV irradiation. The green sample presents very weak ferromagnetism with saturation magnetization of $\sim 7 \times 10^{-5} \mathrm{emu} / \mathrm{g}$. The saturation magnetization for the UV irradiated $\mathrm{BaTiO}_{3}$ crystallites is substantially enhanced and becomes $\sim 6.7 \times 10^{-4} \mathrm{emu} / \mathrm{g}$, due to the increase of oxygen vacancies caused by UV photons. However, the coercive field does not change and remains to be $\sim 305$ Oe. The inset of Fig. 11 is the $M-H$ curve of the sintered bulk sample. The sintered bulk sample is diamagnetic. This behavior is similar to other nanosized oxides particles due to the magnetic origin of defects.
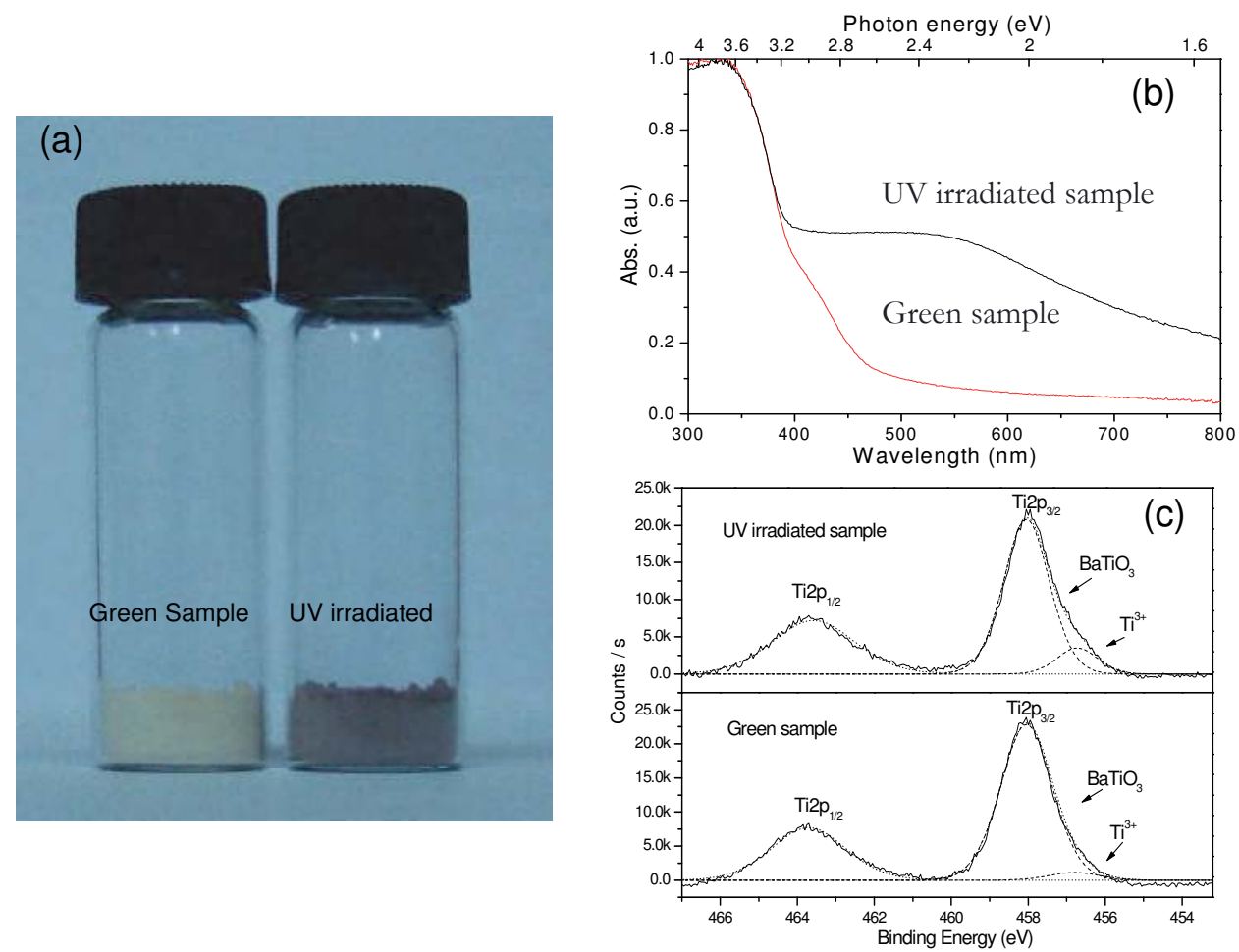

Fig. 10. Photographs of (111) twinned BaTiO3 nanoparticles (a), the corresponding UV-vis absorption spectra (b) and XPS spectra (c) before and after UV irradiation reveal photochromic effect. (Copyright 2010 @ American Chemical Society). 


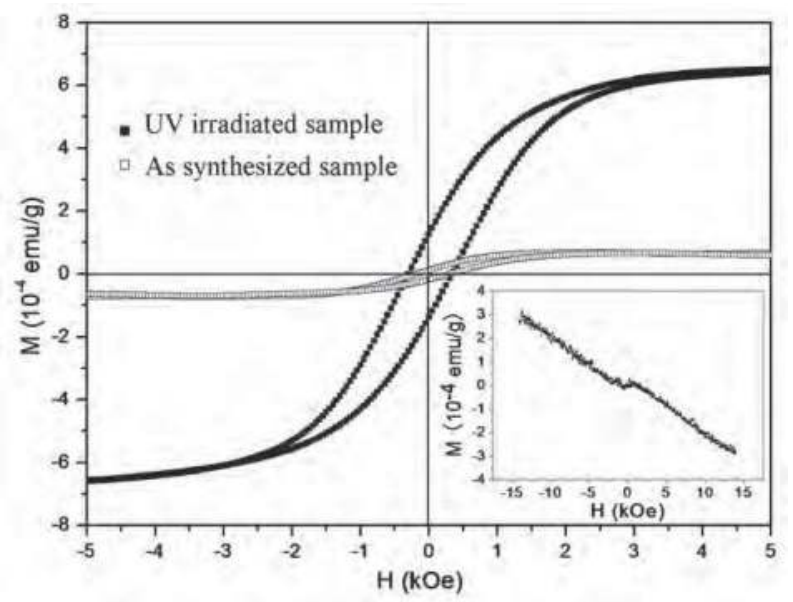

Fig. 11. Room-temperature $\mathrm{M}-\mathrm{H}$ curves of the UV-irradiated $\mathrm{BaTiO}_{3}$ sample and the assynthesized sample. The inset is the M-H curve of the sintered bulk sample. (Copyright 2010 @ American Chemical Society).

\section{Conclusions}

Insightful understanding and careful control of defect structures in ferroelectric does not only provide an efficient tool for tuning ferroelectric properties, but also open a window for exploring novel properties of ferroelectric materials, previously believed impossible or negligible. We expect that there will be more investigations conducted on this area not only from the viewpoint of ferroelectrics but also with cautious consideration of their technical implications.

Acknowledgement. The authors would thank National Science Foundation of China (NSFC) through grant \# 50702031, \# 51021062 and \# 60974117, the Excellent Young Investigators Award Foundation of Shandong Province (Grant No. BS2009CL021), SRF for ROCS, State Education Ministry, National Basic Research Program of China (973 Program) through grant \# 2009CB930503) for financial support.

\section{References}

[1] Scott, J. F. Science. 2007, 315, 954-959.

[2] Damjanovic, D. Rep. Prog. Phys. 1998, 61, 1267-1324.

[3] Gopalan, V.; Dierolf, V. ; Scrymgeour, D. A.;Annu. Rev. Mater. Res., 2007, 37, 449.

[4] Scrymgeour, D. A. ; Gopalan, V. ; Phys. Rev. B, 2005, 72, 24103.

[5] Majdoub, M. S; Sharma, P.; Cagin, T. Phys. Rev. B. 2008, 77, 125424-1-125424-9.

[6] Devonshire, A.F.; Philosophical Magazine, 1949, 40, 1040.

[7] Devonshire, A.F.; Philosophical Magazine, 1951, 42, 1065.

[8] Lines M.E.; Glass, A.M.; Principles and applications of ferroelectrics and related materials [Oxford University Press, New York, 1977].

[9] Abplanalp, M.; Fousek, J.; Gunter, P.; Phys. Rev. Lett., 2001, 86, 5799.

[10] Haun, M.J.; Furman, E.; Jang, S.J.; Mckinstry, H.A.; Cross, L.E.; J. Appl. Phys., 1987, 62, 3331. 
[11] Agullo-Lopez, F.; Catlow, C. R. A.; Townsend, P.; Point Defects in Materials, 1988, Academic Press, London.

[12] Stoneham, A. M. ; Theory of Defects in Solids, 1976, Clarendon Press, Oxford.

[13] Waser, R. ; Smyth, D. M.; Ferroelectric Thin Films: Synthesis and Basic Properties, ed. C. A. P. Araujo, J. F. Scott, 1996, pp. 47-92, GW Taylor, Singapore: Gordon \& Breach.

[14] Jiang, Q. Y.; Subbarao, E. C ; Cross, L. E.; J. Appl. Phys., 1994, 75, 7433.

[15] Jiang, Q. Y. ; Subbarao, E. C. ; Cross, L. E. ; Ferroelectrics, 1994, 154, 119.

[16] Jiang, Q. Y. ; Cao, W. ; Cross, L. E. ; J. Am. Ceram. Soc., 1994, 77, 211.

[17] Duiker, H. M. ; Beale, P. D. ; Scott, J. F. ; Paz de Araujo, C. A. ; Melnick, B. M. ; Cuchiaro, J. D. ; McMillan, L. D. ; J. Appl. Phys., 1990, 68, 5783.

[18] Scott, J. F. ; Dawber, M. ; Appl. Phys. Lett., 2000, 76, 3801.

[19] Ramesh, R. ; Gilchrist, H. ; Sands, T. ; Keramidas, V. G. ; Hakenaasen, R.; Appl. Phys. Lett., 1993, 63, 3592.

[20] Ramesh, R. ; Lee, J. ; Sands, T. ; Keramidas, V. G. ; Auciello, O. ;Appl. Phys. Lett., 1994, 64, 2511.

[21] Chen, H. J. ; Chen, Y. W. ; Ind. Eng. Chem. Res., 2003, 42, 473.

[22] Dutta, P. K. ; Asiaie, R.; Akbar, S. A. ; Zhu, W. ; Chem. Mater., 1994, 6, 1542.

[23] Hennings, D.; Schreinemacher, S. ; J. Euro. Ceram. Soc., 1992, 9, 41.

[24] Wada, S. ; Suzuki, T. ; Noma, T. ;jpn. J. Appl. Phys., 1995, 34, 5368.

[25] Wada, S. ; Suzuki, T. ; Noma, T. ; J. Ceram. Soc. Jpn., 1996, 104, 383.

[26] Kapphan, S.; Weber, G.; Ferroelectrics, 1981, 37, 673.

[27] Wada, S.; Suzuki, T. ; Noma, T. ; J. Ceram. Soc. Jpn., 1995, 103, 1220.

[28] Noma, T. ; Wada, S. ; Yano, M. ; Suzuki, T.; J. Appl. Phys., 1996, 80, 5223.

[29] Jia, C. L. ; Lentzen, M. ; Urban, K. ;Science, 2003, 299, 870.

[30] Namai, Y.; Matsuoka, O.; J. Phys. Chem. B, 2005, 109, 23948.

[31] Hirth J.P.; Lothe, J.; Theory of dislocations [Wiley, New York, ed. 2nd, 1982].

[32] Alpay, S.P.; Misirlioglu, I.B.; Nagarajan, V.; Ramesh, R.; Appl. Phys. Lett., 2004, 85, 2044.

[33] Berlincourt D.; Jaffe, H.; Phys. Rev., 1958, 111, 143.

[34] Bell, A.J.; J. Appl. Phys., 2001, 89, 3907.

[35] Chu, M. W.; Szafraniak, I.; Scholz, R.; Harnagea, C.; Hesse, D.; Alexe, M. ; Gosele, U.; Nat. Mater., 2004, 3, 87.

[36] Jia, C. L.; Mi, S. B.; Urban, K.; Vrejoiu, I.; Alexe, M.; Hesse, D.; Phys. Rev. Lett., 2009, 102, 117601.

[37] Kontsos, A. ; Landis, C. M. ; Int.J.Solids Struct., 2009, 46, 1491.

[38] Li, Y. L. ; Hu, S. Y. ; Choudhury, S.; Baskes, M. I.; Saxena, A.; Lookman, T. ; Jia, Q. X. ; Schlom, D. G.; Chen, L. Q. ; J. Appl. Phys., 2008, 104, 104110.

[39] Betouras, J. J. ; Giovannetti, G. ; J. V. D. Brink, Phys. Rev. Lett., 2007, 98, 257602.

[40] Qin, S.; Liu, D. ; Liu, H. ; Zuo, Z.; J. Phys. Chem. C, 2008, 112, 17171.

[41] Eglitis, R.; Borstel, I. G.; Heifets, E.; Piskunov, S.; Kotomin, E. J. Electroceram., 2006, 16, 289-292.

[42] Clark, I. J. ; Takeuchi, T. ; Ohtori, N. ; Sinclair, D. C. J. Mater. Chem., 1999, 9, 83-91.

[43] Blanco-Lopez, M. C. ; Rand, B. ; Riley, F. L. J. Eur. Ceram. Soc. 1997, 17, 281-287.

[44] Siegel, R. W. Annu. Rev. Mater. Sci. 1991, 21, 559-578.

[45] Madhukar, A.; Lu, S. Y.; Konker, A.; Ho, M.; Hughes, S. M.; Alivisatos, A. P. Nano Lett. 2005, 5, 479-482.

[46] Narayan, J. J. Appl. Phys. 2006, 100, 034309[1]-034309[5].

[47] Gryaznov, V. G.; Polonsky, I. A.; Romanov, A. E.; Trusov L. I. Phys. Rev. B. 1991, 44, 42-46.

[48] Joos, B.; Duesbery, M. S. Phys. Rev. Lett. 1997, 78, 266-269.

[49] Watt, J. P.; Peselnick, L. J. Appl. Phys. 1980, 51, 1525-1531. 
[50] Liu, D.; Chelf, M.; White, K. W. Acta Mater. 2006, 54, 4525-4531.

[51] K.-H. Hellwege, Ed., Landolt-Bornstein: Numerical Data and Functional Relationships in Science and Technology, New Series, Group III, vols. 11 and 18, Berlin: SpringerVerlag, 1979 and 1984.

[52] Colby, B.; Liang, Z.; Wildeson, I.H.; Ewoldt, D.H., Sands, T.D.; Garca, R. E.; Stach, E. A.; Nano Lett., 2010, 10, 1568-1573

[53] Remeika, J. P.; Jackson, W. M. J. Am. Chem. Soc. 1954, 76, 940-941

[54] Tennery, V. J.; Anderson, F. R. J. Appl. Phys. 1959, 29, 755-758.

[55] Recnik, A.;. Kolar, D. J. Am. Ceram. Soc. 1996, 79, 1015-1018.

[56] Hu, K. A.; Hiremath, B. V.; Newnham, R. E. Phase. Transit. 1986, 6, 153-164.

[57] Lee, H. Y.; Kim, J. S. J. Am. Ceram. Soc. 2002, 85, 977-980.

[58] Qin, S; Liu, D.; Zheng, F; Zuo, Z.; Liu H; Xu, X; CrystEngComm, 2010, 12, 3003

[59] Qin, S; Liu, D.; Sang, Y.; Zuo, Z.; Zhang, X.; Zheng, F; Liu H; Xu, X; J. Phys. Chem. Lett. 2010, 1, 238.

[60] Cronemeyer, D. C. Phys. Rev. 1959, 113, 1222.

[61] Berglund, C. N.; Braun, H. J. Phys. Rev. 1967, 164, 790.

[62] Paik, U.; Yeo, J. G.; Lee, M. H.; Hackley, V. A.; Jung, Y. G. Mater. Res. Bull. 2002, 37, 1623.

[63] Renik, A.; Bruley, J.; Mader, W.; Kolar, D.; Rühle, M.; Philosophical Magazine Part B , 1994, 70, 1021-1034. 


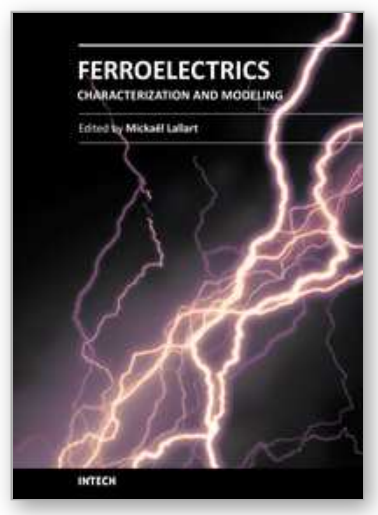

\author{
Ferroelectrics - Characterization and Modeling \\ Edited by Dr. MickaÃ«l Lallart
}

ISBN 978-953-307-455-9

Hard cover, 586 pages

Publisher InTech

Published online 23, August, 2011

Published in print edition August, 2011

Ferroelectric materials have been and still are widely used in many applications, that have moved from sonar towards breakthrough technologies such as memories or optical devices. This book is a part of a four volume collection (covering material aspects, physical effects, characterization and modeling, and applications) and focuses on the characterization of ferroelectric materials, including structural, electrical and multiphysic aspects, as well as innovative techniques for modeling and predicting the performance of these devices using phenomenological approaches and nonlinear methods. Hence, the aim of this book is to provide an up-to-date review of recent scientific findings and recent advances in the field of ferroelectric system characterization and modeling, allowing a deep understanding of ferroelectricity.

\title{
How to reference
}

In order to correctly reference this scholarly work, feel free to copy and paste the following:

Duo Liu (2011). Microstructural Defects in Ferroelectrics and Their Scientific Implications, Ferroelectrics Characterization and Modeling, Dr. MickaÃ«I Lallart (Ed.), ISBN: 978-953-307-455-9, InTech, Available from: http://www.intechopen.com/books/ferroelectrics-characterization-and-modeling/microstructural-defects-inferroelectrics-and-their-scientific-implications

\section{INTECH}

open science | open minds

\section{InTech Europe}

University Campus STeP Ri

Slavka Krautzeka 83/A

51000 Rijeka, Croatia

Phone: +385 (51) 770447

Fax: +385 (51) 686166

www.intechopen.com

\section{InTech China}

Unit 405, Office Block, Hotel Equatorial Shanghai

No.65, Yan An Road (West), Shanghai, 200040, China 中国上海市延安西路65号上海国际贵都大饭店办公楼 405 单元

Phone: +86-21-62489820

Fax: $+86-21-62489821$ 
(C) 2011 The Author(s). Licensee IntechOpen. This chapter is distributed under the terms of the Creative Commons Attribution-NonCommercialShareAlike-3.0 License, which permits use, distribution and reproduction for non-commercial purposes, provided the original is properly cited and derivative works building on this content are distributed under the same license. 